\title{
New developments in multi-meson systems
}

\author{
William Detmold* \\ Thomas Jefferson National Accelerator Facility, 12000 Jefferson Ave, Newpot News, VA 23606, \\ USA \\ Department of Physics, College of William and Mary, Williamsburg, VA 23187, USA \\ E-mail: wdetmoldajlab.org
}

\section{Brian Smigielski}

Department of Physics, National Taiwan University, Taipei, Taiwan

Department of Physics, College of William and Mary, Williamsburg, VA 23187, USA

E-mail: smigs@phys.ntu.edu.tw

New developments in the study of multi-meson systems are reviewed. We highlight a new recursive algorithm for generating the requisite contractions needed for studying complex systems of mesons involving large numbers of particles or multiple species of particles. First results on mixed species systems involving pions and kaons are also presented.

The XXVIII International Symposium on Lattice Field Theory, Lattice2010

June 14-19, 2010

Villasimius, Italy

${ }^{*}$ Speaker. 


\section{Introduction}

Systems containing more than two mesons are of phenomenological interest in a number of areas from heavy ion collisions at RHIC, to the equation of state of neutron stars. In the last few years, there has been a concerted effort by the NPLQCD collaboration to study such systems from first principles using lattice QCD $[1,2,3]$. Much progress has been made and these studies enabled the first calculation of a three hadron interaction, that between three like-charged pions.

On the theoretical side, a number of developments have also occurred in the last few years. First, previous results for the volume dependence of the ground state energies of $n$ boson systems $[4,5,6]$ have been extended to the case of mixed species systems involving pions and kaons [8]. Secondly, a new algorithm has been presented [9] that recursively generates the quark propagator contractions for complicated systems from those of smaller systems. In principle, this method allows the calculation of systems with very large numbers of mesons (surmounting the limit of $n=12$ particles in previous calculations) and of systems involving many different types of bosons. In this contribution, recent aspects of this progress are highlighted.

\section{Multi-Meson Interactions}

It has long been known how to exploit the volume dependence of the eigen-energies of two hadron systems to extract infinite volume scattering phase shifts [10] provided that the effective range of the interaction, $r$ is small compared to the spatial extent of the lattice volume (since $r \sim m_{\pi}^{-1}$ for most interactions, this constraint is equivalent to $m_{\pi} L \gg 1$ ). In recent works, this has been extended to systems involving $n>2$ bosons [4, 5, 6] and $n=3$ fermions [7] in the situation where the relevant scattering length, $a$, is small compared to the spatial extent of the lattice. By performing a perturbative calculation involving two- and three- body hadronic interactions in a finite volume, the ground state energy of a system of $n$ bosons was computed in Refs. [4, 5, 6]. The shift in energy of $n$ bosons of mass $M$ from the non-interacting system is

$$
\begin{aligned}
& \Delta E_{n}=\frac{4 \pi \bar{a}^{n}}{M L^{3}} C_{2}\{1-\left(\frac{\bar{a}}{\pi L}\right) \mathscr{I}+\left(\frac{\bar{a}}{\pi L}\right)^{2}\left[\mathscr{I}^{2}+(2 n-5) \mathscr{J}\right] \\
&-\left(\frac{\bar{a}}{\pi L}\right)^{3}\left[\mathscr{I}^{3}+(2 n-7) \mathscr{I} \mathscr{J}+\left(5 n^{2}-41 n+63\right) \mathscr{K}\right] \\
&+\left(\frac{\bar{a}}{\pi L}\right)^{4}\left[\mathscr{I}^{4}-6 \mathscr{I}^{2} \mathscr{J}+\left(4+n-n^{2}\right) \mathscr{J}^{2}+4\left(27-15 n+n^{2}\right) \mathscr{I} \mathscr{K}\right. \\
&\left.\left.+\left(14 n^{3}-227 n^{2}+919 n-1043\right) \mathscr{L}\right]\right\} \\
&+{ }^{n} C_{3}\left[\frac{192 \bar{a}^{5}}{M \pi^{3} L^{7}}\left(\mathscr{T}+\mathscr{T}_{1} n\right)+\frac{6 \pi \bar{a}^{3}}{M^{3} L^{7}}(n+3) \mathscr{I}\right] \\
&+{ }^{n} C_{3} \frac{1}{L^{6}} \overline{\bar{\eta}}_{3}^{L}+\mathscr{O}\left(L^{-8}\right),
\end{aligned}
$$

where the parameter $\bar{a}$ is related to the scattering length, $a$, and the effective range, $r$, by

$$
a=\bar{a}-\frac{2 \pi}{L^{3}} \bar{a}^{3} r\left(1-\left(\frac{\bar{a}}{\pi L}\right) \mathscr{I}\right) .
$$


The geometric constants, $\mathscr{I}, \mathscr{J}, \mathscr{K}, \mathscr{L}, \mathscr{T}_{0,1}$, that enter into Eq. (2.1) are defined in Ref. [6] and ${ }^{n} C_{m}$ are the binomial coefficients. The three-body contribution to the energy-shift given in Eq. (2.1) is represented by the parameter $\overline{\bar{\eta}}_{3}^{L}$ (see Ref. [6]).

Using lattice QCD calculations of these energy shifts, the parameters $\bar{a}$ and $\overline{\bar{\eta}}_{3}^{L}$ can be extracted (this method was used in Refs. $[1,2,3]$ ). To determine the energy shifts, the multi-meson correlation functions (specifying to the multi-pion system)

$$
C_{n}(t) \propto\left\langle\left(\sum_{\mathbf{x}} \pi^{-}(\mathbf{x}, t)\right)^{n}\left(\pi^{+}(\mathbf{0}, 0)\right)^{n}\right\rangle,
$$

are calculated. On a lattice of infinite temporal extent, ${ }^{1}$ the combination

$$
G_{n}(t) \equiv \frac{C_{n}(t)}{\left[C_{1}(t)\right]^{n}} \stackrel{t \rightarrow \infty}{\longrightarrow} \mathscr{B}_{0}^{(n)} e^{-\Delta E_{n} t}
$$

allows for an extraction of the ground-state energy shift, $\Delta E_{n}$, which can then be used as input into Eq. (2.1) to extract the scattering and interaction parameters. To compute the $(n !)^{2}$ Wick contractions in Eq. (2.3), the correlation function can be written as

$$
\begin{aligned}
C_{n}(t) & \propto\left\langle(\bar{\eta} \Pi \eta)^{n}\right\rangle \propto \varepsilon^{\alpha_{1} \alpha_{2} . . \alpha_{n} \xi_{1} . . \xi_{12-n}} \varepsilon_{\beta_{1} \beta_{2} . . \beta_{n} \xi_{1} . . \xi_{12-n}}(\Pi)_{\alpha_{1}}^{\beta_{1}}(\Pi)_{\alpha_{2}}^{\beta_{2}} . .(\Pi)_{\alpha_{n}}^{\beta_{n}} \\
\Pi & =\sum_{\mathbf{x}} S(\mathbf{x}, t ; 0,0) S^{\dagger}(\mathbf{x}, t ; 0,0)
\end{aligned}
$$

where $S(\mathbf{x}, t ; 0,0)$ is a light-quark propagator. The object (block) $\Pi$ is a $N \times N$ bosonic timedependent matrix where $N=12\left(N=N_{S} \times N_{C}\right.$ with $N_{S}=4$-spin and $N_{C}=3$-color $)$, and $\eta_{\alpha}$ is a twelve component Grassmann variable. Further simplifications are possible resulting in the correlation functions being written in terms of traces of powers of $\Pi$. As an example, the contractions for the $3-\pi^{+}$system give

$$
C_{3}(t) \propto \operatorname{tr}[\Pi]^{3}-3 \operatorname{tr}\left[\Pi^{2}\right] \operatorname{tr}[\Pi]+2 \operatorname{tr}\left[\Pi^{3}\right]
$$

where the traces are over color and spin indices.

\section{Contractions for Large $(N>12)$ Systems of Mesons}

To extend these types of calculations to systems of $n>12$ mesons, or to study systems of many different types of mesons, different methods of performing the contractions of quark fields are required. While better than the naïve factorial construction (which scales as $n !^{2}$ ), the construction used in Section 2 scales poorly to large numbers of mesons, behaving at best as $n !^{1 / 2}$ (provided the matrix $\Pi$ is generalized to give a nonzero result). In Ref. [9], a recursive method for performing these contractions was developed that allows the extension of the study of meson systems to larger $n$ and also greatly simplifies the contractions required for systems of many different species of mesons. We will outline the construction by considering the recursive approach to the contractions for a single species of meson before reporting the general case.

\footnotetext{
${ }^{1}$ Effects of temporal (anti-)periodicity are discussed in Ref. [3].
} 
By rescaling the correlation functions of the single-species, single-source system considered previously as

$$
C_{n \pi^{+}}(t)=(-)^{n} n !\left\langle R_{n}\right\rangle
$$

(the angle brackets denote a trace over spin and color indices) it is straightforward to show that the objects, $R_{n}$ that are defined implicitly in the above equation satisfy an ascending recursion

$$
R_{n+1}=\left\langle R_{n}\right\rangle \Pi-n R_{n} \Pi
$$

with the initial condition that $R_{1}=\Pi$ as defined in Eq. (2.5). To see how this works we explicitly construct the first few terms:

$$
\begin{aligned}
R_{2} & =\left\langle R_{1}\right\rangle \Pi-R_{1} \Pi=\langle\Pi\rangle \Pi-\Pi^{2} \\
\left\langle R_{2}\right\rangle & =\langle\Pi\rangle^{2}-\left\langle\Pi^{2}\right\rangle, \\
R_{3} & =\left\langle R_{2}\right\rangle \Pi-2 R_{2} \Pi=\langle\Pi\rangle^{2} \Pi-\left\langle\Pi^{2}\right\rangle \Pi-2\langle\Pi\rangle \Pi^{2}+2 \Pi^{3} \\
\left\langle R_{3}\right\rangle & =\langle\Pi\rangle^{3}-3\left\langle\Pi^{2}\right\rangle\langle\Pi\rangle-2\left\langle\Pi^{3}\right\rangle,
\end{aligned}
$$

in agreement with Eq. (2.6). Descending recursions also exist.

A correlation function for a system composed of $n_{i j}$ mesons of the $i^{\text {th }}$ species from the $j^{\text {th }}$ source at $\left(\mathbf{y}_{j}, 0\right)$, where $0 \leq i \leq k$ and $0 \leq j \leq m$, is of the form

$$
\begin{aligned}
& C_{\mathbf{n}}(t)=\left\langle\left(\sum_{\mathbf{x}} \mathscr{A}_{1}(\mathbf{x}, t)\right)^{\mathscr{N}_{1}} \ldots\left(\sum_{\mathbf{x}} \mathscr{A}_{k}(\mathbf{x}, t)\right)^{\mathscr{N}_{k}}\right. \\
& \left.\left(\mathscr{A}_{1}^{\dagger}\left(\mathbf{y}_{\mathbf{1}}, 0\right)\right)^{n_{11}} \ldots\left(\mathscr{A}_{1}^{\dagger}\left(\mathbf{y}_{\mathbf{m}}, 0\right)\right)^{n_{1 m}} \ldots\left(\mathscr{A}_{k}^{\dagger}\left(\mathbf{y}_{\mathbf{1}}, 0\right)\right)^{n_{k 1}} \ldots\left(\mathscr{A}_{k}^{\dagger}\left(\mathbf{y}_{\mathbf{m}}, 0\right)\right)^{n_{k m}}\right\rangle,
\end{aligned}
$$

where $\mathscr{N}_{i}=\sum_{j} n_{i j}$ is the total number of mesons of species $i$, and the subscript in $C_{\mathbf{n}}(t)$ labels the number of each species from each source,

$$
\mathbf{n}=\left(\begin{array}{cccc}
n_{11} & n_{12} & \ldots & n_{1 m} \\
\vdots & \vdots & \vdots & \vdots \\
n_{k 1} & n_{k 2} & \ldots & n_{k m}
\end{array}\right)
$$

The $\mathscr{A}_{i}(\mathbf{y}, t)$ denotes a quark-level interpolating operator $\mathscr{A}_{m}(\mathbf{x}, t)=\bar{q}_{m}(\mathbf{x}, t) \gamma_{5} u(\mathbf{x}, t)$, and it can be shown that [9]

$$
C_{\mathbf{n}}(t)=\left(\prod_{i} \mathscr{N}_{i} !\right)\left\langle\prod_{i, j}\left(\bar{\eta} P_{i j} \eta\right)^{n_{i j}}\right\rangle=(-)^{\bar{N}} \frac{\left(\prod_{i} \mathscr{N}_{i} !\right)\left(\prod_{i, j} n_{i j} !\right)}{\mathscr{N} !}\left\langle T_{\mathbf{n}}\right\rangle
$$

where the $\eta$ are $m \times N$-component Grassmann variables, and the $P_{i j}$ are $\bar{N} \times \bar{N}$ dimensional matrices, where $\bar{N}=m \times N$, which are generalizations of the $\Pi$ defined in Eq. (2.5) with an additional 
species index, $i$. They are defined as

$$
\begin{aligned}
P_{i j} & =\left(\begin{array}{c|c|c|c}
0 & 0 & \ldots & 0 \\
\hline \vdots & \vdots & \ldots & \vdots \\
\hline\left(A_{i}\right)_{j 1}(t) & \left(A_{i}\right)_{j 2}(t) & \ldots & \left(A_{i}\right)_{j m}(t) \\
\hline 0 & 0 & \ldots & 0 \\
\hline \vdots & \vdots & \ldots & \vdots \\
\hline 0 & 0 & \ldots & 0
\end{array}\right), \\
\left(A_{i}\right)_{a b} & =\sum_{\mathbf{x}} S\left(\mathbf{x}, t ; \mathbf{y}_{b}, 0\right) S_{i}^{\dagger}\left(\mathbf{x}, t ; \mathbf{y}_{a}, 0\right)
\end{aligned}
$$

The $\left(A_{i}\right)_{a b}$ are $N \times N$ dimensional matrices, one for each flavor, $i$, and pair of source indices, $a$ and $b . \bar{N}=\sum_{i} \mathscr{N}_{i}$ is the total number of mesons in the system, with $\bar{N} \leq \bar{N}$. The $T_{\mathbf{n}}$ defined implicitly in Eq. (3.6) satisfy the recursion relation

$$
T_{\mathbf{n}+\mathbf{1}_{r s}}=\sum_{i=1}^{k} \sum_{j=1}^{m}\left\langle T_{\mathbf{n}+\mathbf{1}_{r s}-\mathbf{1}_{i j}}\right\rangle P_{i j}-\overline{\mathscr{N}} T_{\mathbf{n}+\mathbf{1}_{r s}-\mathbf{1}_{i j}} P_{i j}
$$

where

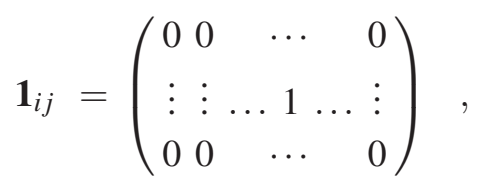

and where the sole non-zero value is in the $(i, j)^{\text {th }}$ entry. Defining $\mathscr{U}_{j}=\sum_{i} n_{i j}$ to be the number of mesons from the $j^{\text {th }}$ source, it is clear that the correlation function vanishes when $\mathscr{U}_{j}>N$ for any source $j$.

These recursion relations (and those for the simpler systems also studied in Ref. [9]) allow for the calculation of arbitrarily large systems of mesons. As formulated above, the systems are restricted to contain quarks of one flavor but anti-quarks of any number of flavors ${ }^{2}$ or vice versa. Importantly the above algorithm scales linearly with the total number of mesons in the system.

This recursive construction has been implemented and is currently being used to study a variety of complex meson systems that were previously not accessible. Generalisations to the case of baryons are straightforward but cumbersome.

\section{Mixed Species Meson Systems}

In the case of a mixed system comprising $n$ mesons of one type and $m$ mesons of a second type, the results summarized in Section 2 have been generalized in Ref. [8], working to $\mathscr{O}\left(1 / L^{6}\right)$. The energy shift of the interacting system from the free system depends on three two-body interaction parameters and four three-body interaction parameters. As might be expected, the full form of the energy shift is lengthy and we refer the interested reader to the original paper.

\footnotetext{
${ }^{2}$ This restriction is not necessary, but relaxing it results in much more complex sets of recursions.
} 
These systems are currently under numerical study and preliminary results using $L^{3} \times T=$ $20^{3} \times 128$ anisotropic clover lattices at a pion mass of $390 \mathrm{MeV}$ generated by the Hadron Spectrum Collaboration are discussed herein.

In order to extract the energies of the multi meson systems, we study the behavior of two point correlation functions

$$
C_{N, M}(t)=\left\langle\left(\sum_{\mathbf{x}} \pi^{-}(\mathbf{x}, \mathbf{t})\right)^{\mathbf{N}}\left(\sum_{\mathbf{x}} \mathbf{K}^{-}(\mathbf{x}, \mathbf{t})\right)^{\mathbf{M}}\left(\pi^{+}(\mathbf{0}, \mathbf{t})\right)^{\mathbf{N}}\left(\mathbf{K}^{+}(\mathbf{0}, \mathbf{t})\right)^{\mathbf{M}}\right\rangle,
$$

where the pion and kaon interpolating operators are defined in terms of quark fields as $\pi^{+}(\mathbf{x}, t)=$ $\bar{u}(\mathbf{x}, t) \gamma_{5} d(\mathbf{x}, t)$ and $K^{+}(\mathbf{x}, t)=\bar{u}(\mathbf{x}, t) \gamma_{5} d(\mathbf{x}, t)$, respectively.

At large Euclidean times, $0 \ll t \ll T$, these correlation functions decay exponentially with the ground state energies, $E_{N, M}$, of the system. These energies could then be used to constrain the twoand three- body interactions using the results of Ref. [8]. However, satisfying the constraint $0 \ll$ $t \ll T$, requires taking the limit of vanishing temperature and is not typically practical in current lattice calculations. The presence of thermal fluctuations that vanish exponentially as $T \rightarrow \infty$, leads, at finite temporal extent, to significantly more complex behaviour of the correlation functions in Eq. (4.1). The general form for these correlation functions can be straightforwardly deduced from Hamiltonian evolution at finite temperature and is given by the following

$$
\begin{aligned}
C_{N, M}(t)= & \frac{1}{2} \sum_{m=0}^{M} \sum_{n=0}^{N} Z_{n, m}^{N-n, M-m} e^{-\left(E_{N-n, M-m}+E_{n, m}\right) T / 2} \cosh \left(\left(E_{N-n, M-m}-E_{n, m}\right)(t-T / 2)\right) \\
& +\frac{1}{2} Z_{\frac{N}{2}, \frac{N}{2}}^{\frac{N}{2}, \frac{M}{2}} e^{\left(E_{N / 2, M / 2}\right) T / 2} \delta_{N, 2 l} \delta_{M, 2 k}+\ldots
\end{aligned}
$$

where the last term is only present when $N$ and $M$ are both even numbers. The ellipsis denotes excited state contributions. The subleading terms shown in the summation correspond to the various ways one can connect the $N$-pion, $M$-kaon source and sink, allowing for propagation of subsets of hadrons around the temporal boundary.

With the large number of parameters that occur in Eq. (4.2), fitting the correlation functions becomes a complicated endeavour. Our current analysis strategy employs variable projection (VarPro $[12,13])$ to eliminate the linear parameters, the $Z_{n, m}^{N-n, M-m}$, from the minimisation procedure. As $N$ and $M$ increase, we also employ the method of Bayesian priors [14] by providing suitable physical starting points for the value of the energies in the correlation function. A summary plot of the energy extractions is shown in Fig. 1.

Analysis of these correlation functions is ongoing and preliminary results are very encouraging. The data provide statistically significant extractions of most of the two- and three- body interactions that enter the mixed-species systems. It additionally allows us to study the relationships between the isospin and hypercharge densities and the corresponding chemical potentials.

\section{Summary}

Systems of large numbers of mesons provide a novel form of QCD matter that is amenable to study using lattice QCD. A rich phase structure is expected and the tools and methodologies presented herein will allow a close study of these systems. These techniques also represent an 


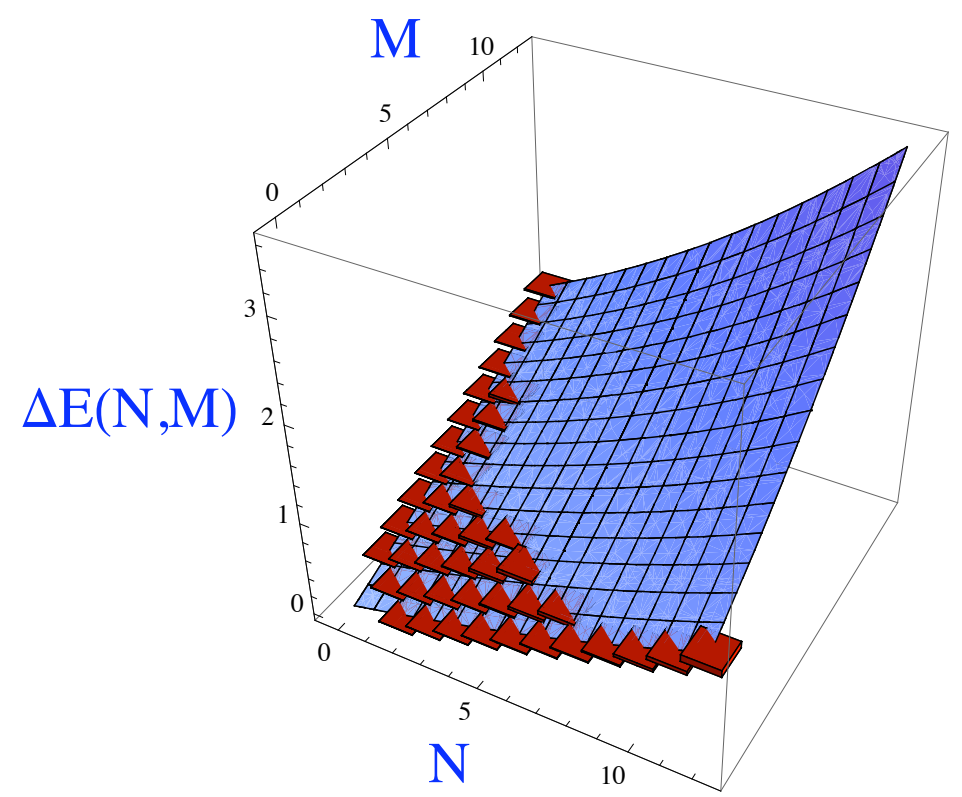

Figure 1: Energies of systems of $N$ pions and $M$ kaons extracted from our calculations. The vertical extent of the shaded boxes corresponds the uncertainty in the determination of the energies.

important step towards the complexity frontier that must be confronted if lattice QCD is to be applied to the physics of nuclei.

\section{References}

[1] S. R. Beane, W. Detmold, T. C. Luu, K. Orginos, M. J. Savage and A. Torok, Phys. Rev. Lett. 100, 082004 (2008) [arXiv:0710.1827 [hep-lat]].

[2] W. Detmold, et al., Phys. Rev. D 78, 014507 (2008).

[3] W. Detmold, K. Orginos, M. J. Savage and A. Walker-Loud, Phys. Rev. D 78, 054514 (2008).

[4] S. R. Beane, W. Detmold and M. J. Savage, Phys. Rev. D 76, 074507 (2007).

[5] S. Tan, arXiv:0709.2530 [cond-mat.stat-mech].

[6] W. Detmold and M. J. Savage, Phys. Rev. D 77, 057502 (2008) [arXiv:0801.0763 [hep-lat]].

[7] T. Luu, PoS LATTICE2008, 246 (2008) [arXiv:0810.2331 [hep-lat]].

[8] B. Smigielski and J. Wasem, Phys. Rev. D 79, 054506 (2009) [arXiv:0811.4392 [hep-lat]].

[9] W. Detmold and M. J. Savage, arXiv:1001.2768 [hep-lat].

[10] M. Lüscher, Commun. Math. Phys. 105, 153 (1986).

[11] W. Detmold and B. Smigielski, in preparation.

[12] L. Kaufman, BIT 15, 49, 1975.

[13] G. T. Fleming, hep-lat/0403023.

[14] G. P. Lepage, et al., Nucl. Phys. Proc. Suppl. 106, 12-20 (2002). [hep-lat/0110175]. 